\title{
RENDRE VISIBLE LE DRAME, FAIRE PENSER LE RELI- GIEUX AUTREMENT : DU RÔLE DE LA COMMUNICATION DigitAle DANS L'ACTION DE L'ONG IMAM ALI EN IRAN
}

\author{
Modjtaba NAJAFI ${ }^{1}$
}

\begin{abstract}
Partant de l'événement du tremblement de terre en Azerbaïdjan de 2012, cet article examine la façon dont l'organisation non gouvernementale Imam Ali tente de rendre visibles les populations rurales marginalisées. En analysant son discours, on engage ici une discussion sur le rôle des témoignages et des images dans la constitution d'un monde commun grâce aux activités collectives menées par cette organisation lors du tremblement de terre. Cet article affirme que cette organisation met à profit une lecture plurale de la religion pour faire reconnaître les victimes et les intégrer dans le cadre englobant d'une communauté éthique. L'article explique la stratégie de visibilité adoptée par Imam Ali, qui aboutit à une lutte pour la reconnaissance des victimes du tremblement de terre, en particulier dans les villages qui étaient hors de l'attention de l'espace public iranien.
\end{abstract}

\section{Introduction}

Les activités de l'organisation non gouvernementale (ONG) Imam Ali lors du séisme d'Azerbaïdjan en Iran en 2012 forment l'objet du

1 Modjtaba NajAfi est docteur en Sciences de l'Information et de la Communication de l'Université Sorbonne nouvelle - Paris 3.

Recherches en communication, $\mathrm{n}^{\circ} 53$ - Article publié le 15/09/2021 
présent article. Imam Ali est l'une des organisations iraniennes engagées pour intervenir face aux difficultés sociales, en particulier le travail des enfants, la pauvreté, l'exécution des mineurs, etc. Créée en 1999 à l'université Sharif à Téhéran, cette organisation regroupe des étudiants iraniens qui veulent rendre visibles les populations marginalisées. Constituant l'une des plus grandes et des plus actives ONG iraniennes, elle est parvenue à développer ses activités dans seize provinces. Imam Ali compte plus de dix mille adhérents dans tout le pays. L'organisation protège près de 6100 enfants en difficulté. Le but d'Imam Ali est annoncé dans sa charte : le renforcement de la participation publique dans les politiques sociales, la relecture des cérémonies et des rites nationaux religieux en vue de promouvoir les conditions sociales et humaines ${ }^{2}$.

Cet article met en évidence l'attitude religieuse d'Imam Ali, qui est tout à fait contre celle du pouvoir. En raison de cette opposition, elle est toujours sous la pression des ultraconservateurs ${ }^{3}$. Au début du mois de mars 2021, cette ONG a d'ailleurs été dissoute par le pouvoir juridique. Imam Ali attend le jugement de la cour d'appel ; pour certains analystes iraniens, cette décision est perçue comme un signe de la sensibilisation du pouvoir à l'égard de l'influence des acteurs indépendants de la société civile.

Imam Ali utilise l'espace public numérique pour sensibiliser l'opinion en diffusant ses textes, ses images et ses vidéos sur les réseaux sociaux comme YouTube et Facebook. L'importance de ce sujet réside dans une piste de recherche qui prend en compte non seulement l'efficacité des réseaux sociaux dans la visibilité des acteurs indépendants et des sans-voix mais également dans le séisme comme une scène d'observation. Le séisme devient un objet social par lequel nous pouvons identifier les nouvelles formes de sociabilité en Iran, les différentes orientations politico-sociales, le niveau de préparation du pouvoir face aux défis qui mettent en danger la sécurité des citoyens. Par le séisme, nous voyons la mobilisation des symboles, des signes, des mythes d'une société. En particulier, dans ce genre d'événements, un grand nombre d'Iraniens ont l'opportunité de s'exprimer car le niveau des risques sécuritaires est moindre que durant des événements politiques

2 Voir ce lien : https://sosapoverty.org/

3 Les critiques des journaux ultraconservateurs et les hashtags des Bassidjis sur Twitter contre l'ONG Imam Ali sont sévères ; voir https://www.bbc.com/persian/iran47932381. 
comme le mouvement Vert en 2009 (Najafi, 2015). Ce caractère favorise la participation de plus en plus importante des citoyens dans les débats. En un mot, par le séisme, où la société est mobilisée dans son ensemble, nous pouvons connaître l'Iran, expliquer les mutations de la société en nous concentrant sur la situation dans laquelle l'événement a eu lieu. En d'autres termes, au cours de l'événement, un monde est détruit et un nouveau monde se forme (Arquembourg, 2011).

Le 12 août 2012, un séisme a eu lieu dans la province de l'Azerbaïdjan oriental, située au nord-ouest de l'Iran. Ce séisme a secoué Ahar, Varzaghan et Harisse. Il a causé la mort de 306 personnes et fait 5000 blessés. Il a touché des régions rurales. En analysant le séisme d'Azerbaïdjan, nous verrons de quelle manière une lecture tolérante de la religion se manifeste à travers les textes, les actions, les interprétations, les débats publics de l'ONG Imam Ali. Comment Imam Ali utilise-t-elle les témoignages et les images pour donner une perspective critique et rendre perceptible l'événement pour les spectateurs? Cet article s'interrogera sur le contexte dans lequel cette ONG a interprété cet événement majeur. Il se demandera également comment cette ONG a tenté de mobiliser ses interlocuteurs en adoptant une stratégie de visibilité en vue de révéler les réalités cachées et ignorées au sein de l'espace public iranien. Cet article interroge donc la narrativité de cette ONG lors du séisme en s'appuyant sur l'analyse de discours. Dans un premier temps, nous allons examiner comment la lecture plurale et tolérante de la religion dans l'espace public a aidé à faire apparaître la nouvelle société civile iranienne, caractérisée par la création de nouvelles ONG dans les années 2000. Dans un second temps, nous analyserons les témoignages et les images d'Imam Ali selon une approche pragmatique du langage qui insiste sur la performativité des énoncés.

Notre méthodologie est l'analyse de discours qui prend en compte la situation sociale dans laquelle un discours se produit (Maingueneau, 2005). Selon cette approche, l'activité langagière apparaît immédiatement sociale. Il y a en effet une unité dialectique entre la linguistique et l'extralinguistique (Guilhaumou, 2002). Pour cette méthodologie « La constitution des événements publics mobilise une quantité d'acteurs, elle est sous-tendue par des réseaux socio-techniques divers, et entre en résonance avec des valeurs, des croyances, des normes et ou des cadres d'interprétation qui sont ceux d'une société donnée, à une époque donnée ; de la sorte, ils font advenir des sujets collectifs, mobilisent des publics, et deviennent une ressource pour des débats publics » (Arquembourg, 2011, p. 161). 
Notre corpus est construit en deux niveaux : un corpus de référence et un corpus de travail. Le premier constitue le contexte global de l'analyse, il a le statut de référentiel représentatif. Le corpus de travail constitue un ensemble des éléments pour lesquels on souhaite obtenir une caractérisation (Rastier \& Pincemin, 1999). Le corpus du travail s'appuie sur une sélection en vue du traitement de notre problématique.

Pour la constitution de notre corpus, nous avons recueilli tous les témoignages et les images partagés sur la page Facebook et sur le site d'information de cette organisation non gouvernementale durant trois mois après le séisme, ce qui a permis d'analyser la posture de celle-ci dans le processus de reconnaissance. Nous avons choisi les témoignages, les images et les vidéos les plus exemplaires, ces éléments permettant de façon suffisante de développer notre réflexion à l'égard des activités de l'ONG Imam Ali.

\section{L'apparition de la nouvelle société civile iranienne}

La révolution islamique en 1979 a été une étape importante de l'histoire iranienne qui a abouti à la domination du clergé conservateur sur le pouvoir (Bazargan, 1984). Ce dernier a pu supprimer ses rivaux : les religieux modérés, les partis de gauche, les nationalistes..., en s'appuyant sur une base populaire puissante. Mais en dépit de l'appropriation absolue du pouvoir par le clergé conservateur, l'idée d'islamisation postrévolutionnaire a échoué (Adelkhah, 1998) dans les années 1990 en raison de profonds changements de fait de la démographie (la jeunesse de l'Iran), de la scolarisation massive (la création de nombreuses universités) et du développement des outils de communication (la presse) (Hourcade, 2010). Durant ces années, une fraction du clergé conservateur est devenue réformatrice (Najafi, 2017). La victoire écrasante de Mohammad Khatami, le président réformiste élu en 1997, est la conséquence de ces évolutions qui marquaient un nouvel Iran postrévolutionnaire. Les années 1997-2005 sont désignées comme la période de la société civile ; elle est marquée par l'apparition du discours de la société civile en Iran (Najafi, 2015). Ce discours a été répandu dans l'espace public par le gouvernement dont le slogan était le développement de la société civile (Namazi, 2000). Les médias réformistes iraniens ont insisté sur la nécessité de créer des organisations non gouvernementales pour aider l'État à résoudre les problèmes sociaux (Mohebi, 2012). Une culture de la citoyenneté s'est alors formée qui s'est concrétisée dans les activités de ces ONG destinées à atténuer les souffrances des popu- 
lations, offrir des services sociaux, lutter contre la pauvreté, la maladie, la délinquance, etc. (Squirre, 2006).

Parallèlement à ces changements profonds dans la société iranienne, nous observons les tentatives des intellectuels religieux ${ }^{4}$ de concilier la religion et la démocratie (Kian, 1997). Ces derniers ont essayé d'articuler la société civile iranienne avec la religion, en soutenant une lecture de l'islam fondée sur le pluralisme et la diversité. À titre d'exemple, Abdolkarim Soroush, une grande figure de l'intelligentsia iranienne, s'est efforcé d'ouvrir une nouvelle voie de l'Islam en proposant une interprétation de la religion compatible avec la démocratie (Mottaghi, 2001). Il considère la diversité et le pluralisme comme des fondements de la société civile. Selon lui, il convient de donner une lecture pluraliste de la religion pour que les deux puissent cohabiter dans la société :

«Une lecture de la religion qui dénie la diversité peut être dangereuse pour la survie de la société civile » (Soroush, 1998, p. 135).

Dans ce cadre, Khosrokhavar confère de l'importance au rôle des intellectuels religieux qui a émergé dans les années 1990 :

« Pour Soroush, on peut penser à une nouvelle société civile islamique qui serait régie comme une démocratie et où le religieux n'interviendrait que par l'adhésion de chacun à un islam spirituel. Les normes politiques seraient "islamiques" dans le sens où les individus au sein de cette société se réclameraient, dans leur for intérieur, de l'islam. Cela garantirait l'autonomie de la société (les normes démocratiques y règneraient) tout en donnant à l'islam une signification éminente, chacun y adhérant de son plein gré et les croyants fondant ainsi un ordre politique non régi

4 Par l'expression « intellectuels religieux » nous regroupons des intellectuels relevant de différentes idéologies qui sont unanimes sur la nécessité de la séparation de la religion du pouvoir séculier. Leur différend renvoie toutefois à la sphère de l'intervention de la religion dans l'espace public. L'organisation Imam Ali est sous l'influence d'un discours qui donne de l'importance à la religion comme une forme de communication et de renforcement des principes moraux de la société. 
par une transcendance quelconque » (Khosrokhavar, 2008, p. 354).

Nous pouvons prendre en compte les caractéristiques communes des intellectuels religieux qui ont défendu la réconciliation de la religion et de la société civile. Comme Khosrokhavar le montre :

« Les nouveaux intellectuels religieux se caractérisent par la volonté de contester la légitimité religieuse de la théocratie chiite et plus généralement, de l'islamisme radical, leur but étant de promouvoir une version du religieux qui justifie l'ouverture de la scène politique au pluralisme » (Ibid., p. 351).

À côté des intellectuels, nous observons les tentatives du clergé réformiste, qui n'est pas le courant dominant dans les écoles religieuses, mais on entend une voix qui invite le pouvoir islamique à réformer la relation entre la religion et l'État. Pour beaucoup de chercheurs, le clergé a joué le rôle d'une institution civique indépendante du pouvoir dans l'histoire contemporaine iranienne (Kamali, 2003). Après la révolution islamique, le statut du clergé a radicalement changé et sa relation avec le système politique s'est resserrée. Le clergé iranien a perdu son indépendance avec la création du gouvernement islamique (Najafi, 2019). Malgré cela, le clergé réformateur a essayé de donner une lecture réformiste de l'islam (Kian, 1995). Certains des ayatollahs réformateurs comme Aliakbar Sanei ont lancé des fatwas pour défendre les droits des femmes, la réconciliation des lois religieuses et civiques. Mohsen Kadivar a écrit deux ouvrages qui refusaient la théorie du Velayath Fagih (Kadivar, 1997). Selon lui, il n'y a pas de consensus parmi les Ulémas chiites sur cette théorie comme fondement théorique de la théologie islamique. Certains refusent la nécessité de l'État islamique et tous les types de théocratie (Kadivar, 1998).

Nous pouvons dire que les intellectuels religieux et le clergé réformateur ont remis en cause la légitimité de la théocratie en défendant la société civile. Tous les intellectuels religieux de toutes tendances sont unanimes sur les deux principes : la légitimité du pouvoir vient du peuple non pas de Dieu, c'est-à-dire qu'un pouvoir sans l'adhésion des citoyens est illégitime ; l'existence de la société civile non seulement

5 Voir ce lien : https://tinyurl.com/3cat7mra 
n'est pas en contradiction avec l'islam mais aussi elle peut garantir une religion plus ouverte et plus dynamique.

\subsection{Le séisme et la lecture humaniste de la religion}

Parallèlement aux débats, présentés au sein de l'espace public, nous observons comment une lecture tolérante et humaniste de la religion se manifeste lors du séisme de l'Azerbaïdjan oriental, région située au nord-ouest de l'Iran. L'insistance des intellectuels religieux iraniens sur la tolérance et la liberté humaine face à un type de déterminisme a opposé les lectures réformistes de l'islam aux conservatrices (Kian, 1997). Cette lecture est « l'Islam Rahmani », c'est-à-dire un islam fondé sur l'amitié de Dieu, qui met en question les idéologies rigoureuses. Celles-ci ont été renforcées depuis la révolution 1979 lorsque la théorie de la « guidance du juriste » (wilâyat al-faqîh) a été adoptée dans la constitution (Arminjon, 2010). La lecture conservatrice établit une relation entre les catastrophes naturelles et l'indignation de Dieu envers les hommes, en estimant que ces catastrophes sont des châtiments divins contre les pécheurs ${ }^{6}$. Le séisme d'Azerbaïdjan a créé une opportunité pour défier cette croyance. Une grande partie des posts des internautes iraniens refuse d'attribuer ces catastrophes à Dieu en insistant sur la volonté libre des humains et l'importance de la méthode scientifique dans la gestion du pays (Najafi, 2019). Par ailleurs, « la théologie du séisme » se manifeste lors de cet événement : si elle critique la lecture religieuse traditionnelle et explique qu'il faut croire en la générosité de Dieu, qu'il faut s'appuyer sur sa puissance, ces convictions ne sont cependant pas contradictoires avec la surveillance de la construction, de la consolidation, de l'utilisation des bons matériaux pour les bâtiments...

Dans ce cadre, non seulement les citoyens internautes mais également les ONG actives dans ce séisme ont défié la lecture conservatrice de l'islam. Nous voyons comment une narration indépendante du pouvoir se forme dans l'espace public. Ce ne sont pas seulement les

6 Mohammad Bagherian, le savant religieux conservateur rattaché à l'institut de Dar $\mathrm{Al}$ Hadith se réfère au hadith du sixième imam des chiites qui fait le lien entre l'adultère et la survenue du séisme. Disponible sur https://tinyurl.com/8htxp25w Ailleurs, Kazem Sedighi, le prêcheur nommé par le guide suprême, met en relation le séisme et certains péchés comme l'adultère et le non-respect du voile. https://www.bbc. com/persian/iran/2010/05/100506_119_earthquake_women 
médias officiels qui présentent leur narration à propos du séisme mais également les citoyens internautes et les ONG qui ont participé à la constitution d'une narration indépendante de celle du pouvoir. Cette narration a pour but la reconnaissance des victimes comme des sujets sociaux qui ont leurs propres droits dans l'espace public.

Dans ce contexte, les citoyens internautes et les ONG dans leur présence sur les réseaux sociaux ne se sont pas limités à partager l'information mais ils ont tenté de communiquer à travers leurs expériences et leurs efforts pour encourager les lecteurs à entreprendre une action collective. En effet, l'Iran est entré dans une nouvelle ère grâce à l'élargissement de l'espace public sous l'influence du développement de l'internet et des réseaux sociaux. Cette évolution a favorisé la création d'un espace pour l'intervention d'acteurs de plus en plus nombreux au sein de la société civile. Cette évolution peut s'expliquer par « les changements dans la pratique de l'internet ». Selon Dominique Cardon (2010), ces changements sont liés au phénomène de massification et de personnalisation qui ont abouti au «tournant du web 2.0 ». Ce changement majeur se caractérise par «l'importance de la participation des utilisateurs à la production des contenus et par leur mise en relation » (Cardon, 2011, p .141).

\section{Les témoignages : rendre perceptible l'événement}

La parole et l'action du témoin dans les reportages et les récits permettent de mettre en scène les expériences du malheur d'autrui(Lavocat, 2010). En effet, Imam Ali met en œuvre la stratégie de médiatisation de la souffrance des victimes du tremblement de terre, ce qui lui permet de faire reconnaître les victimes et aussi de mieux se faire connaître. Les formes narratives tissent le lien entre les expériences du tremblement de terre et les réalités sociales iraniennes. Selon le processus d'identification d'un problème social est repérable parmi des activités qui : " [...] ponctuent le déroulement de la vie sociale, certaines occurrences, situations ou pratiques deviennent "problématiques" et acquièrent le statut médiatique de "faits notables", dignes d'un traitement spécifique et d'une mise en récit structurée »(Voirol, 2005, p. 101).

La visibilité médiatisée de cette organisation rend perceptible l'événement, l'objective dans des textes, des sons et des images. Elle construit un univers moral-pratique par son regard théologique particulier sur les réalités du tremblement de terre (Ibid.). Les témoignages introduisent la manière de voir de l'organisation à travers la situa- 
tion singulière du séisme. Par ces témoignages, prend forme le regard d'Imam Ali. Ce regard est donc marqué par une focale située dans la construction du visible, qui d'après Olivier Voirol : " s'adresse à un public imaginé physiquement absent » (Ibid., p. 99).

À ce titre, nous avons analysé un reportage publié dans le site d'information de cette organisation, partagé aussi sur Facebook; il décrit la situation des victimes du tremblement de terre afin d'encourager les citoyens à se mobiliser pour les aider. Dans ce reportage, nous constatons que cette organisation établit un lien entre les zones défavorisées et la gestion par le gouvernement du tremblement de terre. Le reportage montre que ces populations sont hors de l'attention des organes gouvernementaux : «En effet, quelques jours après le séisme, ces populations sont encore oubliées 7 . » Ensuite, il explique les actions menées par l'ONG pour identifier les villages oubliés par les médias officiels. Le reportage a un accent critique ; il ne s'arrête pas au présent et il rappelle la pauvreté de ces zones en précisant que, même dans les catastrophes naturelles, elles ont été oubliées et négligées par le gouvernement : «Notre équipe de trois personnes a identifié les besoins des populations marginalisées d'Ahar. Ces zones étaient déjà défavorisées et nous imaginons qu'elles doivent toujours l'être y compris dans les situations de crise comme le tremblement de terre ${ }^{8}$. »

Le reportage nous montre les efforts de l'ONG Imam Ali pour faire reconnaitre les victimes du tremblement de terre, en particulier les habitants des zones marginalisées, en supposant que la visibilité est la condition de la reconnaissance (Honneth, 2004). En effet, Imam Ali tente d'interpréter la réalité sociale de la pauvreté et le mépris dans lequel sont tenues les populations marginalisées pour mener une lutte pour la reconnaissance (Honneth, 1992).

\subsection{La religion peut servir au progrès social}

L'organisation Imam Ali possède une dimension religieuse, présente dans sa charte, éloignée de celle des conservateurs. Autrement dit, cette organisation s'appuie sur une éthique qui insiste sur les responsabilités des citoyens à l'égard des crises sociales. Selon elle, les croyants

7 Disponible sur https://www.facebook.com/sosapoverty/photos/a.115504098468793 /475099672509232/?type $=3 \&$ theater

8 Disponible sur https://www.facebook.com/sosapoverty/photos/a.115504098468793 /475099672509232/?type=3\&theater 
doivent s'engager à aider les pauvres et les populations marginalisées. Par conséquent, les devoirs religieux ne se limitent pas aux pratiques de la charia, ils s'étendent à l'engagement des citoyens dans les activités sociales. En effet, Imam Ali préconise une théologie de la libération. Cette dernière critique l'islam ultra-orthodoxe qui prône une approche littérale de la religion. Elle estime que la religion peut servir au progrès social et peut être active au sein de la société civile. D'après cette théologie, les croyants doivent prendre en compte les réalités sociales pour servir les pauvres et les sans-abris ; en ce sens, combattre l'injustice devient, s'il ne l'est déjà, un devoir religieux ${ }^{9}$.

Dans les témoignages partagés, nous constatons les effets de cette théologie dans les points de vue et les actions des volontaires. Ils récusent cette idée selon laquelle le tremblement de terre a des causes métaphysiques. Les conservateurs ont historiquement lié les tremblements de terre à une faute et une sanction, ils donnent une dimension religieuse à l'événement. Mais dans la théologie d'Imam Ali, les citoyens sont responsables de leurs actes, ils doivent consolider les bâtiments, et Dieu ne veut pas que les humains soient dans le malheur. Nous ne pouvons pas, estiment-ils, attribuer nos fautes à Dieu.

En ce sens, Morteza Keymanesh invite ses lecteurs à réfléchir. Il mentionne un hadith attribué à l'imam Ali, le premier imam des chiites, qui estime qu' « une heure de réflexion est mieux que 70 ans de prière ${ }^{10} »$. Ce volontaire pose la question : «Pourquoi attribuons-nous nos crises à Dieu? Il ne faut pas oublier que chacun d'entre nous a une part dans le bien et le mal de ce monde... Lorsque les malheurs se propagent, on fait appel à Dieu et on lui demande pour quels péchés, il nous punit. Nous attribuons les pensées, les paroles et les actes de la partie noire de notre être à Dieu ${ }^{11}$ !» Enfin, il pose une autre question : «Les tremblements sociaux qui fragilisent les fondements de la justice, de la liberté et de notre amour ne sont-ils pas le résultat de la fin de notre pensée ? »

Ces témoignages vont au-delà de la description et s'adressent aux sentiments des lecteurs. Les populations rurales et les enfants sont au centre de l'attention et des actions de l'organisation. Suivant la théologie de la libération, les valeurs partagées entre les Iraniens appellent à voir les réalités concernant les populations défavorisées. La médiatisation de la souffrance des victimes du tremblement de terre s'inscrit dans

9 Voir la charte de cette association disponible sur https://sosapoverty.org/en/

10 Disponible sur https://sosapoverty.org/journal/goleyakh8.pdf

$11 \mathrm{Ibid}$. 
le cadre de la lutte d'Imam Ali pour la reconnaissance des invisibles de la société iranienne. Cette lutte pour la reconnaissance des victimes du séisme se manifeste sous forme de la théologie qui donne de l'importance à la justice en vue de révéler les réalités cachées et ignorées à la société iranienne. Nous voyons comment cette organisation non gouvernementale essaie de partager sa théologie en publiant non seulement des témoignages mais également des images.

\subsection{Les images mobilisent les citoyens}

Pour analyser les images, nous nous appuierons sur l'approche pragmatique du langage qui prend l'image comme un signe (Dewey, 1993). Selon Dewey, l'image fait partie du langage. En reliant une définition large du langage à la théorie de l'acte de parole d'Austin et à la sémiotique de Peirce qui insiste sur les valeurs symboliques, indicielles et iconiques du signe, nous pouvons parler de l'action de l'image dans ce sens que l'image n'est pas sur le mode constatif, elle fait une action (Arquembourg, 2010). Cette approche considère la performativité des images et prend en compte la révélation médiatique comme un acte illocutoire et ses effets sur le destinataire comme les actes perlocutoires (Ibid.). Les images produites et partagées par Imam Ali s'inscrivent dans le schéma de stratégie de la visibilité ; elles montrent non seulement l'événement du tremblement de terre, mais aussi le problème social des populations marginalisées qui sont en dehors de l'espace public iranien. En particulier, les images permettent de positionner cette organisation comme un acteur reconnu menant des expériences communes qui débouchent sur la construction d'une vie éthique. Cette vie éthique se traduit par une compréhension pratique dans laquelle l'acteur partage non seulement les valeurs morales, mais aussi les objectifs éthiques des autres membres de la société. De cette manière-là, les images invitent le public iranien à reconnaître les villageois et les enfants comme des citoyens iraniens à part entière qui ont des droits spécifiques dans 1'espace public.

Une image montre les pratiques d'Imam Ali dans la construction des maisons ${ }^{12}$ : «Aujourd'hui, les villages d'Ahar et de Varzaghan ont tremblé. Notre organisation est en train de construire des maisons pour les victimes. \) Les commentaires de l'image insistent sur la solidarité

12 Disponible sur https://www.facebook.com/sosapoverty/photos/a.115504098468793 $/ 480573908628475 /$ ?type $=3 \&$ theater 
en utilisant les concepts religieux : « Des jeunes plein d'amour et de foi se sont consacrés au peuple de leur pays, ils apprennent jour et nuit les méthodes de construction de maisons solides. » Ensuite, nous voyons le partage des croyances religieuses appelant à la bienfaisance dans une structure symbolique qui invite les spectateurs à la résistance contre les catastrophes : «De nouveau, les villes ont tremblé mais la maison de l'amour et du sacrifice de nos jeunes ne tremblera pas. »

Les légendes de ces images partagent l'émotion et cette dernière est une forme de communication (Nussbaum, 1995). Imam Ali partage l'amour, la foi, le sacrifice qui montrent son attitude religieuse à l'égard de cet événement et par lesquels l'ONG invite ainsi ses interlocuteurs à construire un public solidaire face à la catastrophe naturelle.

Nous observons comment les légendes encouragent les spectateurs à voir de près la réalité du tremblement de terre et les actions que mène l'organisation : « venez voir de près ». Le dernier énoncé est performatif dans ce sens qu'il encourage ses destinataires à reconstruire les régions détruites.

Dans une autre image, nous observons un enfant dans les débris ${ }^{13}$. Nous voyons ce titre : " Jouer pour les enfants, c'est la vie ». Ensuite, des énoncés à portée affective sont utilisés afin de partager les émotions : « Il y a plusieurs jours que l'on n'entend que leurs gémissements. Est-ce qu'il y a quelqu'un qui peut leur rendre leurs bons souvenirs de jeu ? "La maison du paradis des enfants" attend votre aide. "

Dans les images partagées, les victimes et les décombres constituent le visible et proposent un dispositif de visibilité. Comme Jacques Rancière le souligne dans Le Spectateur émancipé : «Le dispositif de visibilité règle le statut des corps représentés et le type d'attention qu'ils méritent. La question est de savoir le type d'attention que provoque tel ou tel dispositif» (2008, p. 108).

Le dispositif concentre l'attention sur la pauvreté des enfants et des paysans dans les régions frappées par le tremblement de terre. Il donne du sens à la réalité sociale en Azerbaïdjan devenue visible par l'événement du séisme et rendue perceptible par Imam Ali. Jacques Rancière pose que l'image est seulement un élément dans un dispositif qui produit le sens de la réalité, un sens commun constitué de données sensibles (Ibid.). Il insiste aussi sur le rôle des mots. Selon lui, les mots

13 Disponible sur https://www.facebook.com/sosapoverty/photos/a.115504098468793 /474037652615434/?type=3\&theater, la page consultée le 10/10/2018. 
ne sont pas à la place des images, ils sont des images, c'est-à-dire des formes de redistribution des éléments de la représentation (Ibid.).

\subsection{Les messagers de Dieu sur la terre}

Imam Ali ne se limite pas aux images fixes mais elle partage également des images mobiles sur les réseaux sociaux. Dans un clip partagé sur YouTube ${ }^{14}$, cette organisation non gouvernementale a regroupé les grands artistes iraniens qui présentent leurs condoléances aux victimes du tremblement de terre en Azerbaïdjan. Dans ce clip réalisé dans « La maison iranienne du centre du soutien aux enfants travailleurs », les grands cinéastes iraniens interviennent à côté des « enfants du travail » pour parler non seulement des conséquences épouvantables du tremblement de terre sur les enfants, les pauvres gens, les sans-abris, mais aussi pour discuter des crises sociales en Iran et de leur impact. Cette vidéo commence par les images du séisme en Azerbaïdjan et la présence des volontaires de cette organisation au côté des populations, notamment des enfants et des sans-abris. Ensuite commencent les messages des artistes. Les artistes expliquent l'impact de l'événement sur les enfants qui ont perdu leurs parents dans le tremblement de terre. Peu à peu, le débat prend une dimension plus publique. « La vie continue », « Il faut donner l'espoir à ceux qui ont été sauvés des ruines », « N'ayez pas peur, ne soyez pas inquiet Dieu est plus grand », tels sont les messages transmis par les artistes et les « enfants du travail ». Les récepteurs de ces messages ne sont pas précisés : s'agit-il des enfants d'Azerbaïdjan, victimes du tremblement de terre qui ont perdu leurs parents ? Ou des Iraniens qui regardent le clip ? Puis, le débat se généralise : les artistes relient les problèmes publics de la société iranienne à ce tremblement de terre. Les débats vont au-delà du séisme en Azerbaïdjan et les intervenants orientent l'attention sur les crises sociales au sein de la société iranienne. Nous voyons l'attitude religieuse d'Imam Ali dans cette vidéo. L'un des artistes appelle les enfants « les messagers de Dieu sur la terre » : « Je crois que tous les enfants sont les messagers de Dieu sur la terre, ils sont innocents. Si nous les oublions, demain ils deviendront des délinquants. '»

En montrant les images des enfants travailleurs en Iran, les intervenants expliquent les deux tremblements de terre qui ont affecté le pays : un tremblement de terre naturel - comme celui de Bam en 2003

14 Disponible sur : https:/www.youtube.com/watch?v=fKGV-XzOIuE 
et d'Azerbaïdjan en 2012 - et un séisme social, qui est en cours au cœur de la société iranienne. Ici apparaît une métaphore dans le terme de tremblement de terre. Il se traduit en surface par des vibrations du sol, il provient de la fracturation des roches en profondeur. Mais, dans le second sens, le séisme a lieu dans la profondeur de la société : c'est le séisme généré par les crises sociales iraniennes dont l'une des plus importantes est liée à la situation défavorable des « enfants du travail ».

Dans l'une des séquences de ce clip, les enfants travailleurs parlent de leurs expériences de lavage des vitres des voitures. L'un des enfants parle de la mort de ses parents. Il compare la perte de ses parents à celle des enfants pris par le tremblement de terre afin d'amener ses interlocuteurs à la profondeur de la douleur des « enfants du travail ». Dans ce clip, nous constatons aussi comment les cinéastes iraniens relient la question des enfants frappés par le tremblement de terre à celle, plus importante au niveau national, des « enfants du travail » en Iran.

Ce clip partagé sur YouTube en septembre 2012 appelle à une solidarité avec les sinistrés du tremblement de terre plus particulièrement les enfants. Les artistes iraniens invitent leurs compatriotes à être ensemble ; les énoncés sont courts et enchaînés, ils portent une certitude. En effet, chaque artiste est responsable de l'énoncé d'une phrase : " La mère, le père et la famille, qui peut être soudainement désintégrée », "Chacun d'entre eux rêve, dans les décombres du tremblement de terre », " Peut-être demande-t-il la mort ; la maison qui s'écroule », " Peut-être la nourriture est-elle la dernière préoccupation de l'enfant », «Peut-être l'enfant attend-il une marque d'affection, une voix et un regard d'amitié $» . .$.

Les énoncés sont performatifs et affectueux, ils s'expriment dans une scène sentimentale accompagnée d'une musique traditionnelle iranienne. Le tremblement de terre a été une opportunité pour Imam Ali afin d'ouvrir des débats sur les problèmes publics comme ceux du travail des enfants, de la pauvreté, des populations marginalisées. L'utilisation des énoncés performatifs et émotifs est une forme de communication qui peut diminuer la distance entre le spectateur et les victimes.

Dans l'imagerie partagée par Imam Ali, nous voyons des formes de représentation compatibles avec les missions énoncées dans la charte, plus particulièrement le combat contre la pauvreté, l'aide aux enfants défavorisés et aux populations marginalisées. Toute action est soumise à une opération de sélection. Les images, les témoignages obéissent à cette opération afin de décrire les réalités sociales choisies par cette organisation. Les commentaires de la légende dans l'image vont 
au-delà de la description de l'événement. Ils invitent le public iranien à la solidarité. « Les mains aidantes et les yeux attendus », « Nos mains ne sont pas vides », « Nous avons emprunté le fardeau de l'amour », « Nous mettons les mains dans les mains », « Nous allumons nos maisons avec la lumière de l'espoir » ont un caractère poétique, émouvant et religieux, qui proposent une forme de solidarité pour susciter des pratiques communes.

\section{Conclusion}

Les résultats de notre analyse montrent comment une catastrophe naturelle peut être une opportunité pour établir des relations entre les acteurs d'un événement et les victimes. Ainsi, les expériences vécues des volontaires de l'ONG Imam Ali et des populations villageoises d'Azerbaïdjan sont capables de bouleverser les frontières de la visibilité et de l'invisibilité. C'est par la stratégie de la visibilité menée par cette organisation que les victimes sont reconnues. Cette stratégie consiste à révéler les réalités qui jusque-là étaient ignorées ou cachées. La mise en œuvre de cette stratégie a nécessité d'orienter l'attention publique vers les populations marginalisées dans les villages des régions frappées par le séisme. La reconnaissance des victimes s'est concrétisée au fil des textes, des récits, des témoignages, et plus particulièrement des images fixes et animées. Sans cette visibilité, le processus de la reconnaissance est impossible. Cette $\mathrm{ONG}$ a réussi à établir une relation avec un public à travers ses témoignages lors d'une opération de reconnaissance des victimes. Nous voyons également que dans le tremblement de terre en Azerbaïdjan, une lecture tolérante de la religion se manifeste dans 1'espace public iranien. On y reconnaît les populations marginalisées et le problème public de l'injustice. En s'appuyant sur une lecture plurale de la religion, l'organisation Imam Ali a adopté une stratégie de visibilité, qui aboutit à une lutte pour la reconnaissance des victimes du tremblement de terre en particulier dans les villages qui étaient hors de l'attention de l'espace public iranien.

\section{Références}

Adelkhah, F. (1998). Etre moderne en Iran. Paris : Karthala.

Arminjon, C. (2010). L'instauration de la " guidance du juriste » en Iran. Archives de sciences sociales des religions, 149, 211-228. Doi : https://doi.org/10.4000/ assr.21941 
Arquembourg J. (2010). Des images en action. Performativité et espace public. Réseaux, 163(5), 163-187. Doi : https://doi.org/10.3917/res.163.0163

Arquembourg, J. (2011). L'Événement et les médias. Les récits médiatiques des tsunamis et les débats publics (1755-2004). Paris : Archives contemporaines.

Bazargan, M. (1984). Enghelabe Iran dar do harkat, [La révolution d'Iran en deux étapes]. Téhéran : Bonyad Farhangi Mohandes Bazargan.

Cardon, D. (2010). La Démocratie internet. Promesses et limites. Coll. La République des idées. Paris : Seuil.

Cardon, D. (2011). Réseaux sociaux de l'Internet. Communications, 88(1), 141-148. Doi : https://doi.org/10.3917/commu.088.0141

Dewey, J. (1993). Logique. La théorie de l'enquête. Paris : PUF.

Guilhaumou, J. (2002). Le corpus en analyse de discours : perspective historique, Corpus, 1. Doi : https://doi.org/10.4000/corpus.8

Honneth, A. (1992). La Lutte pour la reconnaissance. Paris : Cerf.

Honneth, A. (2004). Visibilité et invisibilité. Sur l'épistémologie de la « reconnaissance». Revue du MAUSS, 23(1), 137-151. Doi : https://doi.org/10.3917/ rdm.023.0137

Hourcade, B. (2010). Géopolitique de l'Iran. Paris : Armand Colin.

Kadivar, M. (1997). Nazriate Dolat dar Figh Chiya, [Les théories du gouvernement dans le fiqh de chiisme (la jurisprudence islamique)]. Téhéran : Nashr Ney.

Kadivar, M. (1998). Hokomat Velayi, [Gouvernement de Velayat Fagih]. Téhéran : Nashr Ney.

Kamali, M. (2003). Do enghelab Iran, Modernité, Jameye Madani va mobareze Tabaghati, [Deux révolutions de l'Iran, La modernité, la société civile et les luttes de classes]. Téhéran : Digar.

Khosrokhavar, F. (2008). Les nouveaux intellectuels en Iran. Cahiers internationaux de sociologie, 125(2), 347-363. Doi : https://doi.org/10.3917/cis.125.0347

Kian, A. (1995), L'invasion culturelle occidentale : mythe ou réalité ? Cahiers d'études sur la Méditerranée orientale et le monde turco-iranien, 20. Doi : https://doi. org/10.4000/cemoti.1668

Kian, A. (1997). Stratégies des intellectuels religieux et clercs iraniens face à la modernité occidentale. Revue française de science politique, 47(6),776-797.

Lavocat, F. (2010). Témoignage et récit de catastrophe. Cahiers du dix-septième siècle, 13(1), 32-48.

Maingueneau, D. (2005). L'analyse du discours et ses frontières. Marges linguistiques, 9. Disponible à http://dominique.maingueneau.pagesperso-orange.fr/pdf/L-analysedu-discours-et-ses-frontieres.pdf

Mohebi, M. (2012). Intellectuals, Reform and the Making of a Modern Iranian Civil Society (1997-2005), A thesis submitted to the Department of Social Policy of the London School of Economics for the Degree of Doctor of Philosophy. Disponible à http://etheses.1se.ac.uk/537

Mottaghi, M. (2001). Rowshanfekran e eslahtalab e dini va jameeyemadani [Les intellectuels réformateurs religieux et la société civile]. Iran Nameh, 4, 493-520.

Najafi, M. (2015). La Face féminine du mouvement Vert iranien de l'internet à la rue. Paris : L'Harmattan.

Najafi, M. (2017). Sheikhe Pragmatiste, biographi Siasi ye Mehdi Karoubi[Pragmatist Cleric: A Political Biography of Mahdi Karoubi]. Londres : Éditions Crescent. 
Najafi, M. (2019). L'espace public solidaire face aux séismes de Bam et d'Azerbaïdjan en 2003 et en 2012 : de l'Iran civil à l'Iran des réseaux, thèse sous la direction de Jocelyne Arquembourg, Université Sorbonne nouvelle Paris 3.

Namazi, B. (2000). Non Governmental Organisations in the Islamic Republic in Iran: A Situation Analysis, United nations development programme, Tehran.

Nussbaum, M. (1995). Les émotions comme jugements de valeur. Dans P. Paperman \& R. Ogien (éds), La couleur des pensées, sentiments, émotions, intentions. Coll. Raisons pratiques, 6 (pp. 19-32). Paris : EHESS.

Rancière, J. (2008). Le Spectateur émancipé. Paris : La Fabrique.

Rastier, F. \& Pincemin, B. (1999). Des genres à l'intertexte. Cahiers de praxématique, 33, 83-111. Doi : https://doi.org/10.4000/praxematique.1974

Soroush, A. (1998). Dine va jameye madani, [La religion et la société civile]. Dans Jameye Madani va Iran e Emrooz [La société civile et l'Iran d'aujourd'hui], Téhéran : Éditions Nagsh o Neghr.

Squire, C. (2006), Praxis Paper 8: Building Organisational Capacity in Iranian Civil Society: Mapping the Progress of CSOs [Civil Society Organizations], Intrac.

Voirol, O. (2005). Les luttes pour la visibilité. Esquisse d'une problématique. Réseaux, 129-130, 89-121. Doi : https://doi.org/10.3917/res.193.0043 


\section{(c) (1) (8) $\odot$}

«Attibution - pas d'utilisation Commerciale - Pas de Modification 4.0. International» (CC BY-NC-ND) 